\title{
ON COMPANION INEQUALITIES RELATED TO HEINIG'S
}

\author{
GOU-SHENG YANG AND YU-JEN LIN
} \begin{abstract}
A number of companion inequalities related to Heinig's and their dis-
crete analogues are investigated.
\end{abstract}
\section{Introduction.}

In [1], Heinig established the following three inequalities:

Theorem $\mathbb{A}$. Let $p, s, \lambda$ be real numbers satisfying $p+s>\lambda, p>0$. If

$$
\int_{0}^{\infty} t^{\lambda-s}|f(t)| d t<\infty
$$

then,

$$
\int_{0}^{\infty} x^{\lambda} \exp \left[p x^{-p} \int_{0}^{x} t^{p-1} \log \left|x^{-s} f(t)\right| d t\right] d x \leq e^{1 / p} A \int_{0}^{\infty} t^{\lambda-s}|f(t)| d t
$$

where $A=p /(p+s-\lambda)$.

Theorem $\mathbb{B}$. Let $2 p-1>\lambda-s p, p>0$ and

$$
\int_{0}^{\infty} t^{\lambda-s p}|f(t)|^{p} d t<\infty
$$

Then

$$
\int_{0}^{\infty} x^{\lambda} \exp \left[p^{2} x^{-p} \int_{0}^{x} t^{p-1} \log \left|x^{-s} f(t)\right| d t\right] d x \leq e B \int_{0}^{\infty} t^{\lambda-s p}|f(t)|^{p} d t
$$

where $B=p /(2 p+s p-\lambda-1)$. Theorem C. Suppose $\left\{a_{n}\right\}_{n=1}^{\infty}$ is a non-negative sequence and $s>0, p \geq 1,0 \leq$
$\lambda<s+p$, If $\sum_{n=1}^{\infty} n^{\lambda-s} a_{n}=M<\infty$,

Received June 22, 1990. 
then

$$
\sum_{n=1}^{\infty} n^{\lambda-s p} \prod\left(a_{n}, p\right) \leq e^{1 / p} C \sum_{n=1}^{\infty} n^{\lambda-s} a_{n}
$$

where $\prod\left(a_{n}, p\right)=\left[a_{1} a_{2}^{2^{p-1}} \cdots a_{n}^{n^{p-1}}\right]^{p / n^{p}}, n=1,2,3, \cdots$, and $C=p[1+1 /(p+s-\lambda)]$.

The following improvement of the inequalities (1), (2) and (3) are given by Cochran and Lee [3].

Theorem $\mathbb{D}$. Let $p, r$ be real numbers with $p>0$. If

$$
\int_{0}^{\infty} t^{r}|f(t)| d t<\infty
$$

then

$$
\int_{0}^{\infty} x^{r} \exp \left[p x^{-p} \int_{0}^{x} t^{p-1} \log |f(t)| d t\right] d x \leq e^{(r+1) / p} \int_{0}^{\infty} t^{r}|f(t)| d t .
$$

Theorem $\mathbb{E}$. Let $p, r$ be real numbers with $p \geq 1, r \geq 0$. If $\left\{a_{n}\right\}_{n=1}^{\infty}$ is a sequence of non-negative real numbers less then or equal to unity with $\sum_{n=1}^{\infty} n^{r} a_{n}<\infty$, then

$$
\sum_{n=1}^{\infty} n^{r} \prod\left(a_{n}, p\right) \leq e^{(r+1) / p} \sum_{n=1}^{\infty} n^{r} a_{n}
$$

The purpose of this paper is to establish some companion inequalities that are related to inequalities (1)-(5).

II. Companiom inequalities related to Heinig's.

Theorem. 1. Let $p, s, \lambda$ be real numbers satisfying $p+s<\lambda, p<0$, If

$$
\int_{0}^{\infty} t^{\lambda-s}|f(t)| d t<\infty
$$

then

$$
\int_{0}^{\infty} x^{\lambda} \exp \left[-p x^{-p} \int_{x}^{\infty} t^{p-1} \log \left|x^{-s} f(t)\right| d t\right] d x \leq e^{1 / p} A \int_{0}^{\infty} t^{\lambda-s}|f(t)| d t,
$$

where $A=p /(p+s-\lambda)$.

Proof. Since

$$
e^{1 / p}=\exp \left[p \int_{1}^{\infty} y^{p-1} \log y d y\right]
$$


a change of variable shows that (6) has the form

$$
\begin{aligned}
& \int_{0}^{\infty} x^{\lambda} \exp \left[-p x^{-p} \int_{1}^{\infty} x^{p-1} y^{p-1} \log \left|x^{-s} f(x y)\right| x d y\right] d x \\
\leq & e^{1 / p} A \int_{0}^{\infty} t^{\lambda-s}|f(t)| d t
\end{aligned}
$$

which is equivalent to

$$
\int_{0}^{\infty} x^{\lambda} \exp \left[-p \int_{1}^{\infty} y^{p-1}\left(\log \left|x^{-s} y f(x y)\right|\right) d y\right] d x \leq A \int_{0}^{\infty} t^{\lambda-s}|f(t)| d t .
$$

But by Jensen's inequality ([5], p.62) the left side of the above inequality is dominated by

$$
\begin{aligned}
& -p \int_{0}^{\infty} x^{\lambda}\left[\int_{1}^{\infty}\left|x^{-s} y^{p} f(x y)\right| d y\right] d x \\
= & -p \int_{1}^{\infty} y^{p+s-\lambda-1}\left[\int_{0}^{\infty} t^{\lambda-s}|f(t)| d t\right] d y \\
= & A \int_{0}^{\infty} t^{\lambda-s}|f(t)| d t
\end{aligned}
$$

which is obtained by an interchange of order of integration which is justified by Fubini's Theorem.

Theorem 2. Let $2 p-1<\lambda-s p, p<0$ and

$$
\int_{0}^{\infty} t^{\lambda-s p}|f(t)|^{p} d t<\infty
$$

Then

$$
\begin{aligned}
& \int_{0}^{\infty} x^{\lambda} \exp \left[-p^{2} x^{-p} \int_{x}^{\infty} t^{p-1} \log \left|x^{-s} f(t)\right| d t\right] d x \\
\leq & e B \int_{0}^{\infty} t^{\lambda-s p}|f(t)|^{p} d t
\end{aligned}
$$

where $B=p /(2 p+s p-\lambda-1)$.

Proof. Since

$$
e=\exp \left[p^{2} \int_{1}^{\infty} y^{p-1} \log y d y\right]
$$

a change of variable shows that (7) has the form

$$
\begin{aligned}
& \int_{0}^{\infty} x^{\lambda} \exp \left[-p^{2} x^{-p} \int_{1}^{\infty} x^{p-1} y^{p-1} \log \left|x^{-s} f(x y)\right| x d y\right] d x \\
\leq & e B \int_{0}^{\infty} t^{\lambda-s p}|f(t)|^{p} d t
\end{aligned}
$$


which is equivalent to

$$
\int_{0}^{\infty} x^{\lambda} \exp \left[-p^{2} \int_{1}^{\infty} y^{p-1} \log \left|x^{-s} y f(x y)\right| d y\right] d x \leq B \int_{0}^{\infty} t^{\lambda-s p}|f(t)|^{p} d t
$$

By Jensen's inequality the left side of the above inequality is dominted by

$$
\begin{aligned}
& \int_{0}^{\infty} x^{\lambda}\left[\int_{1}^{\infty}\left|x^{-s} y f(x y)\right|^{p}(-p) y^{p-1} d y\right] d x \\
& =-p \int_{1}^{\infty} y^{2 p-1}\left[\int_{0}^{\infty} x^{\lambda-s p}|f(x y)|^{p} d x\right] d y \\
& =B \int_{0}^{\infty} t^{\lambda-s p}|f(t)|^{p} d t
\end{aligned}
$$

which is obtained by an interchang of order of integration which is justified by Fubini's Theorem.

For the discrete analogue of theorem 1 it is convenient to introduce the following notation. Let $\left\{a_{n}\right\}_{n=1}^{\infty}$ be a sequence of non-negative real numbers and $p<0$. Then we write

$$
Q\left(a_{n}, p\right)=\left[a_{n}^{n-1} a_{n+1}^{(n+1)^{p-1}} \cdots\right]^{-p / n^{p}}, n=1,2,3, \cdots
$$

Theorem 3. Suppose $\left\{a_{n}\right\}_{n=1}^{\infty}$ is a non-negative sequence and $s \leq 0, p \leq-1$, $\lambda \geq 0$. If $\sum_{n=1}^{\infty} n^{\lambda-s} a_{n}=M<\infty$,

then

$$
\sum_{n=1}^{\infty} n^{\lambda-s p} Q\left(a_{n}, p\right) \leq e^{1 / p} C \sum_{n=1}^{\infty} n^{\lambda-s} a_{n}
$$

where $C=-p[1+1 /(\lambda-p-s)]$.

Proof. Without loss of generality, we may assume that

$$
n^{-s} a_{k} \leq 1, k=n, n+1, \cdots
$$

If $0<M \leq 1$, this is obvious.

If $M>1$, then divide both side of (8) by $M$ to obtain

$$
\frac{1}{M} \sum_{n=1}^{\infty} n^{\lambda-s p} Q\left(\frac{a_{n}}{M}, p\right) M^{\alpha} \leq e^{1 / p} C \sum_{n=1}^{\infty} n^{\lambda-s} \frac{a_{n}}{M}
$$

where $\alpha=\left(-p \sum_{k=n}^{\infty} k^{p-1}\right) / n^{p} \geq 1 \geq p$.

It follows that

$$
\frac{1}{M} \sum_{n=1}^{\infty} n^{\lambda-s p} Q\left(\frac{a_{n}}{M}, p\right) \leq e^{1 / p} C \sum_{n=1}^{\infty} n^{\lambda-s} \frac{a_{n}}{M}
$$


Replacing $a_{n} / M$ by $a_{n}$, we obtain $n^{\lambda-s} a_{n} \leq 1$ and hence $n^{-s} a_{n} \leq n^{-\lambda} \leq 1$ which implies $n^{-s} a_{k} \leq 1, k=n, n+1, \cdots$.

Now to prove (8), observe that

$$
\begin{aligned}
& \sum_{n=1}^{\infty} n^{\lambda-s p} Q\left(a_{n}, p\right) \\
& \leq \sum_{n=1}^{\infty} n^{\lambda+\frac{s p}{n^{p}} \Sigma_{k=n}^{\infty} k^{p-1}} Q\left(a_{n}, p\right) \\
& =\sum_{n=1}^{\infty} n^{\lambda}\left[n^{-s n^{p-1}} n^{-s(n+1)^{p-1}} \cdots\right]^{\frac{-p}{n^{p}}}\left[a_{n}^{n p-1} a_{n+1}^{(n+1)^{p-1}} \cdots\right]^{\frac{-p}{n^{p}}} \\
& =\sum_{n=1}^{\infty} n^{\lambda} \exp \left[\frac{-p}{n^{p}} \sum_{k=n}^{\infty} k^{p-1} \log \left(n^{-s} a_{k}\right)\right] \\
& =\sum_{n=1}^{\infty} n^{\lambda} \exp \left[\frac{-p}{n^{p}} \sum_{k=n}^{\infty} k^{p-1} \log \left(n^{-s} a_{k}\right)\right] \\
& =\sum_{n=1}^{\infty} n^{\lambda} \exp \left\{\frac{-p}{n^{p}} \sum_{k=n}^{\infty} k^{p-1} \int_{k}^{k+1} \log \left[n^{-s} f(t)\right] d t\right\}
\end{aligned}
$$

where

$$
f(t)= \begin{cases}a_{k}, & k<t \leq k+1 \\ 0, & \text { otherwise }\end{cases}
$$

But since $n^{-s} f(t) \leq 1$ the last equality is dominated by

$$
\begin{aligned}
& \sum_{n=1}^{\infty} n^{\lambda} \exp \left\{\frac{-p}{n^{p}} \sum_{k=n}^{\infty} \int_{k}^{k+1} t^{p-1} \log \left[n^{-s} f(t)\right] d t\right\} \\
= & \sum_{n=1}^{\infty} n^{\lambda} \exp \left\{\frac{-p}{n^{p}} \int_{n}^{\infty} t^{p-1} \log \left[n^{-s} f(t)\right] d t\right\} \\
= & \sum_{n=1}^{\infty} n^{\lambda} \exp \left\{-p \int_{1}^{\infty} y^{p-1} \log \left[n^{-s} f(n y)\right] d y\right\},
\end{aligned}
$$

which is less than or equal to $C e^{1 / p} \sum_{n=1}^{\infty} n^{\lambda-s} a_{n}$ if, and only if

$$
\sum_{n=1}^{\infty} n^{\lambda} \exp \left\{-p \int_{1}^{\infty} y^{p-1} \log \left[n^{-s} y f(n y)\right] d y\right\} \leq C \sum_{n=1}^{\infty} n^{\lambda-s} a_{n}
$$


By Jensen's inequality, the left side of (9) is dominated by

$$
\begin{aligned}
& \sum_{n=1}^{\infty} n^{\lambda}\left[-p \int_{1}^{\infty} y^{p} n^{-s} f(n y) d y\right] \\
& =\sum_{n=1}^{\infty} n^{\lambda}\left[-p \int_{n}^{\infty} t^{p} n^{-p-s-1} f(t) d t\right] \\
& =-p \sum_{n=1}^{\infty} n^{\lambda-p-s-1} \sum_{k=n}^{\infty} a_{k} \int_{k}^{k+1} t^{p} d t \\
& \leq-p \sum_{n=1}^{\infty} n^{\lambda-p-s-1} \sum_{k=n}^{\infty} a_{k} k^{p} \\
& =-p \sum_{k=1}^{\infty} k^{p} a_{k} \sum_{n=1}^{\infty} n^{\lambda-s-p-1} \\
& =-p \sum_{k=1}^{\infty} k^{p} a_{k}\left[\sum_{n=1}^{k-1} n^{\lambda-s-p-1}+k^{\lambda-s-p-1}\right] \\
& \leq-p \sum_{k=1}^{\infty} k^{p} a_{k}\left[\int_{0}^{k} t^{\lambda-s-p-1} d t+k^{\lambda-s-p-1}\right] \\
& =-p \sum_{k=1}^{\infty} k^{p} a_{k}\left[k^{\lambda-s-p}\left(\frac{1}{\lambda-s-p}+\frac{1}{k}\right)\right] \\
& \leq-p \sum_{k=1}^{\infty} k^{p} a_{k}\left[k^{\lambda-s-p}\left(\frac{1}{\lambda-s-p}+1\right)\right] \\
& =-p\left(1+\frac{1}{\lambda-s-p}\right) \sum_{n=1}^{\infty} n^{\lambda-s} a_{n} \text {. } \\
& =C \sum_{n=1}^{\infty} n^{\lambda-s} a_{n} \text {. }
\end{aligned}
$$

This completes the proof of this theorem.

The following theorem has been proved in [4], here, we give a simple proof which is motivated in part after [3]:

Theorem 4. Let $p, \beta$ be real numbers with $p<0$. If $\int_{0}^{\infty} t^{\beta}|f(t)| d t<\infty$ and $\int_{0}^{\infty} t^{p-1} \log |f(t)| d t<\infty$

then

$$
\int_{0}^{\infty} x^{\beta} \exp \left[-p x^{-p} \int_{x}^{\infty} t^{p-1} \log |f(t)| d t\right] d x . \leq \exp \left(\frac{\beta+1}{p}\right) \int_{0}^{\infty} x^{\beta}|f(x)| d x
$$


Proof. Consider the following reslt of Hardy ([2], p.246):

If $q>1, r<1$ and $F(x)=\int_{x}^{\infty}|g(t)| d t$, then

$$
\int_{0}^{\infty} x^{-r} F^{q} d x<\left(\frac{q}{1-r}\right)^{q} \int_{0}^{\infty} x^{-r}(x|g|)^{q} d x
$$

Let $\alpha=1-r>0$. Then

$$
\int_{0}^{\infty} x^{\alpha-1}\left[\int_{x}^{\infty}|g(t)| d t\right]^{q} d x<\left(\frac{q}{\alpha}\right)^{q} \int_{0}^{\infty} x^{q+\alpha-1}|g|^{q} d x .
$$

Let $g(x)=x^{p-1} f^{1 / q}$ and set $\beta=p q+\alpha-1$. Then

$$
\int_{0}^{\infty} x^{\alpha-1}\left[-p \int_{x}^{\infty} t^{p-1}|f(t)|^{1 / q} d t\right]^{q} d x<\left(\frac{-p q}{-p q+\beta+1}\right)^{q} \int_{0}^{\infty} x^{q+\alpha-1} x^{p q-q}|f| d x
$$

so that

$$
\int_{0}^{\infty} x^{\beta}\left[-p x^{-p} \int_{x}^{\infty} t^{p-1}|f(t)|^{1 / q} d t\right]^{q} d x<\left(1-\frac{\beta+1}{p q}\right)^{-q} \int_{0}^{\infty} x^{\beta}|f| d x
$$

The desired inequality is obtained by taking the limit $q \longrightarrow \infty$.

Remark 1 . If we set $\lambda-s=\beta$, then the left-side of the inequality (6) becomes the left-side of the inequality (10). And the right-side of inequality (6) is equal to

$$
e^{1 / p}\left(\frac{p}{p-\beta} \int_{0}^{\infty} t^{\beta}|f(t)| d t\right.
$$

Since

for $\lambda-s=\beta>p$, except $\beta=0$.

$$
e^{(\beta+1) / p}<e^{1 / p}\left(\frac{p}{p-\beta}\right)
$$

This shows that the inequality (6) can not be sharp in general and theorem 4 represents an improvement of theorem 1 .

Remark 2. If we set $\lambda-s p=\beta$ and replaced $|f|^{p}$ by $|f|$, then the left-side of the inequality (7) becomes the left-side of the inequality (10) and the right-side of (7) is equal to

Since

$$
e[p /(2 p-\beta-1)] \int_{0}^{\infty} t^{\beta}|f(t)| d t
$$

$$
e^{(\beta+1) / p}<e[p /(2 p-\beta-1)]
$$

for $\beta>2 p-1$, except $\beta=p-1$. 
This shows that the inequality (7) can not be sharp in general and theorem 4 represents an improvement of theorem 2.

Remark 3. When $f(t)=t^{b-\beta} e^{-t^{-p / 2}}$, with $b>-1,(10)$ gives rise to

$$
e^{(\beta-b) / p} 2^{2(b+1) / p} \int_{0}^{\infty} x^{b} e^{-x^{-p / 2}} d x \leq e^{(\beta+1) / p} \int_{0}^{\infty} x^{b} e^{-x^{-p / 2}} d x .
$$

By letting $b \longrightarrow-1$, we readily deduce that the multiplicative constant appearing on the right-hand side of (10) must be best possible.

To prove the finally theorem, which is the principle result in the discrete case, we need the following:

Lemmg. If $0 \leq s<1$ and $0 \leq s+\alpha \leq 1$, then

$$
\left[(i+1)^{s+\alpha}-i^{s+\alpha}\right] \sum_{n=1}^{i} n^{-s} \leq i^{\alpha} \frac{s+\alpha}{1-s}, \quad i=1,2,3, \cdots
$$

Proof. Since

$$
\sum_{n=1}^{i} n^{-s} \leq \int_{0}^{i} x^{-s} d x=\frac{i^{1-s}}{1-s}
$$

it follows from mean value theorem that

$$
\begin{aligned}
& {\left[(i+1)^{s+\alpha}-i^{s+\alpha}\right] \sum_{n=1}^{i} n^{-s} } \\
\leq & {\left[(i+1)^{s+\alpha}-i^{s+\alpha}\right] \frac{i^{1-s}}{1-s} } \\
= & (s+\alpha) C^{s+\alpha-1} \frac{i^{1-s}}{1-s}, \quad i<C<i+1 \\
\leq & (s+\alpha) i^{s+\alpha-1} \frac{i^{1-s}}{1-s} \\
= & \frac{s+\alpha}{1-s} i^{\alpha} .
\end{aligned}
$$

Theorem 5. Let $p, r$ be real numbers with $p \leq-1,0 \leq r \leq 1$. If $\left\{a_{n}\right\}_{n=1}^{\infty}$ is a sequence such that $0 \leq a_{n} \leq 1 . \forall n$ and $\sum_{n=1}^{\infty} n^{r} a_{n}<\infty$,
then

$$
\sum_{n=1}^{\infty} n^{r} Q\left(a_{n}, p\right) \leq(-p) e^{(r-p) / p} \sum_{n=1}^{\infty} n^{r} a_{n}
$$

where

$$
Q\left(a_{n}, p\right)=\left[a_{n}^{n-1} a_{n+1}^{(n+1)^{p-1}} \ldots\right]^{-p / n^{p}}, n=1,2,3, \ldots
$$


Proof. Our demonstration is modelled in part after [3]. Since $0 \leq a_{n} \leq 1$,

$$
\begin{aligned}
& \sum_{n=1}^{\infty} n^{r} Q\left(a_{n}, p\right) \\
= & \sum_{n=1}^{\infty} n^{r} \exp \left[-p n^{-p} \sum_{i=n}^{\infty} i^{p-1} \log a_{i}\right] \\
= & \sum_{n=1}^{\infty} n^{r} \exp \left[-p n^{-p} \sum_{i=n}^{\infty} \int_{i}^{i+1} i^{p-1} \log f(t) d t\right] \\
\leq & \sum_{n=1}^{\infty} n^{r} \exp \left[-p n^{-p} \sum_{i=n}^{\infty} \int_{i}^{i+1} t^{p-1} \log f(t) d t\right] \\
= & \sum_{n=1}^{\infty} n^{r} \exp \left[-p n^{-p} \int_{n}^{\infty} t^{p-1} \log f(t) d t\right] \\
= & e^{(r-p+s) / p} \sum_{n=1}^{\infty} n^{r} \exp \left[-p \int_{1}^{\infty} y^{p-1} \log f(n y) d y\right] \\
& \exp \left[-p \int_{1}^{\infty} y^{p-1} \log y^{r-p+s} d y\right] \\
= & e^{(r-p+s) / p} \sum_{n=1}^{\infty} n^{r} \exp \left\{-p \int_{1}^{\infty} y^{p-1} \log \left[y^{r-p+s} f(n y)\right] d y\right\},
\end{aligned}
$$

where

$$
f(t)= \begin{cases}a_{i}, & i<t \leq i+1, i=n, n+1, \cdots \\ 0, & \text { otherwise. }\end{cases}
$$

and $s$ is chosen so that $0 \leq s<1$, and $0 \leq s+r \leq 1$. By Jensen's inequality, the final summation is dominated by

$$
I=-p \sum_{n=1}^{\infty} n^{r} \int_{1}^{\infty} f(n y) y^{r+s-1} d y
$$


and for this equality, we have

$$
\begin{aligned}
& I=-p \sum_{n=1}^{\infty} n^{r} \int_{n}^{\infty} f(t) t^{r+s-1} n^{-r-s+1} n^{-1} d t \\
& =-p \sum_{n=1}^{\infty} n^{-s} \int_{n}^{\infty} f(t) t^{r+s-1} d t \\
& =-p \sum_{n=1}^{\infty} n^{-s} \sum_{i=n}^{\infty} a_{i} \int_{i}^{i+1} t^{r+s-1} d t \\
& =\frac{-p}{r+s} \sum_{n=1}^{\infty} n^{-s} \sum_{i=n}^{\infty} a_{i}\left[(i+1)^{r+s}-i^{r+s}\right] \\
& =\frac{-p}{r+s} \sum_{i=n}^{\infty} a_{i}\left[(i+1)^{r+s}-i^{r+s}\right] \sum_{n=1}^{i} n^{-s} \\
& \leq \frac{-p}{r+s} \sum_{i=1}^{\infty} a_{i} i^{r} \frac{r+s}{1-s} \quad \text { (by Lemma) } \\
& =\frac{-p}{1-s} \sum_{i=1}^{\infty} a_{i} i^{r}
\end{aligned}
$$

for $0 \leq s<1$, and $0 \leq s+r \leq 1$, by virtue of the definition of $f(t)$ and the previous lemma. The proof is completed by noting that

where the minimum occurs for $s=0$.

$$
-p e^{(r-p) / p}=\min _{s}\left[\frac{-p}{1-s} e^{(r-p+s) / p}\right]
$$

Remark 4. Since

$$
e^{(r-p) / p} \leq e^{1 / p}\{1+[1 /(r-p)]\} \quad \text { for } \quad p \leq-1,0 \leq r \leq 1 .
$$

If we set $r=\lambda-s$, then our inequality $(\mathbb{1} 1)$ of theorem 5 represents a substantial improvement over ( 8 ) of theorem 3 in case $\lambda-s \leq 1$.

\section{References}

[1] H. P. Heining, "Some extensions of Hardy's inequality", SIAM J. Math. Anal 6, 698-713, 1975.

[2] G. H. Hardy, J. E. Littlewood and G. Pólya, "inequalities" Cambridge, 1934.

[3] J. A. Cochran and C. S. Lee, "Inequalities rellated to Hardy's and Heinig's",

Philos, Soc. 96, 1-7, 1984. [4] E. R. Love, "Inequalities related to those of Hardy and of Cochran and Lee", Math. proc. Comb.
Phil. Soc. 99, 395-408, 1986 .

[5] Walter Rudin, "Real and Complex Analysis", Third Edition Mcgraw-Hill book company. 